\title{
Reasons and Risk Factors for Readmission Following Hospitalization for Community-acquired Pneumonia in South Korea
}

\author{
Jong Geol Jang, M.D. ${ }^{(10}$ and June Hong Ahn, M.D., Ph.D. ${ }^{10}$ \\ Department of Internal Medicine, Yeungnam University Medical Center, Yeungnam University College of Medicine, Daegu, \\ Korea
}

Background: Limited studies have been performed to assess readmission following hospitalization for communityacquired pneumonia (CAP) in an Asian population. We evaluated the rates, reasons, and risk factors for 30-day readmission following hospitalization for CAP in the general adult population of Korea.

Methods: We performed a retrospective observational study of 1,021 patients with CAP hospitalized at Yeungnam University from March 2012 to February 2014. The primary end point was all-cause hospital readmission within 30 days following discharge after the initial hospitalization. Hospital readmission was classified as pneumonia-related or pneumonia-unrelated readmission.

Results: During the study period, 862 patients who survived to hospital discharge were eligible for inclusion and among them $72(8.4 \%)$ were rehospitalized within 30 days. In the multivariable analysis, pneumonia-related readmission was associated with para/hemiplegia, malignancy, pneumonia severity index class $\geq 4$ and clinical instability $\geq 1$ at hospital discharge. Comorbidities such as chronic lung disease and chronic kidney disease, treatment failure, and decompensation of comorbidities were associated with the pneumonia-unrelated 30-day readmission rate.

Conclusion: Rehospitalizations within 30 days following discharge were frequent among patients with CAP. The risk factors for pneumonia-related and -unrelated readmission were different. Aspiration prevention, discharge at the optimal time, and close monitoring of comorbidities may reduce the frequency of readmission among patients with CAP.

Keywords: Causes; Hospital Readmission; Koreans; Pneumonia; Risk Factors

Address for correspondence: June Hong Ahn, M.D., Ph.D.

Division of Pulmonology and Allergy, Department of Internal Medicine, Yeungnam University Medical Center, Yeungnam University College of Medicine, 170 Hyeonchung-ro, Nam-gu, Daegu 42415, Korea

Phone: 82-53-640-6577, Fax: 82-53-620-3849

E-mail: fireajh@gmail.com

Received: Oct. 15, 2019

Revised: Nov. 14, 2019

Accepted: Dec. 6, 2019

Published online: Mar. 10, 2020

(c) It is identical to the Creative Commons Attribution Non-Commercial License (http://creativecommons.org/licenses/by-nc/4.0/). The Korean Academy of Tuberculosis and Respiratory Diseases.

\section{Introduction}

Pneumonia is an important cause of morbidity and mortality worldwide. It is the seventh-leading cause of death in the United States, where about 915,900 episodes of pneumonia occur annually in adults aged $\geq 65$ years ${ }^{1}$. In Korea, pneumonia ranked tenth (7.1 deaths per 100,000 population) among all causes of death in 2004 and sixth (21.4 deaths per 100,000 population) in $2013^{2}$. The incidence and direct medical costs of hospitalized pneumonia are consistently high in Korea. Persons aged $\geq 75$ years, and those with underlying medical conditions, are at increased risk of hospitalization due to pneumonia ${ }^{3}$.

Pneumonia is a frequent cause of rehospitalization after discharge; generalized vulnerability to illness also contributes to the development of acute conditions requiring rehospitaliza- 
tion ${ }^{4}$.

The 30-day readmission after hospitalization for pneumonia is related to the rate of 30-day mortality after discharge ${ }^{5}$. Moreover, pneumonia readmission rates are associated with increased medical costs and are used as an indicator of quality of care ${ }^{6}$. Patient-, disease-, physician-, and healthcare systemrelated factors are associated with pneumonia readmissions and are targets of interventions to reduce the rate of readmission for pneumonia ${ }^{7}$. Clinically, identification of the reasons and risk factors for readmission is important for discharge planning.

The rates, reasons, and risk factors for pneumonia readmission vary among populations, regions, and methodologies. Most prior readmission studies were conducted in North America and Europe. One Korean study assessed only healthcare system-related factors in elderly (age $\geq 65$ years) pneumonia patients using nationwide data ${ }^{6}$. Although pneumonia is a frequent cause of readmission, which is related to several modifiable factors, few Asian studies have investigated hospital readmission for pneumonia. Here we evaluated the rates, reasons, and risk factors for 30-day readmission following hospitalization for community-acquired pneumonia (CAP) in the general adult population of Korea.

\section{Materials and Methods}

\section{Study design and subjects}

We performed a retrospective observational study of 1,021 patients with CAP hospitalized at Yeungnam University Hospital (a 930-bed, university-affiliated, tertiary referral hospital in Daegu, Korea) from March 2012 to February 2014. The total number of patients enrolled in this study does not differ from previous publication and this study is a secondary analysis of previous publication using same database ${ }^{8}$. In this study, we added health insurance status as an independent factor. However, we could not find health insurance status in 10 patients based on electronic medical records. Thus, we excluded these patients first.

As described in detail previously ${ }^{8}$, during the study period, all consecutive adult patients (age $\geq 18$ years) with CAP admitted to the hospital via the emergency or outpatient department were eligible for inclusion. Patients with hospitalacquired pneumonia that developed more than 48 hours after admission, those aged below 18 years, immunocompromised patients (such as those with neutropenia after chemotherapy, human immunodeficiency virus infection, solid organ transplant recipients, or receiving corticosteroids or other immunosuppressive agents), patients with active Mycobacteria tuberculosis infection, and patients registered twice were not included in this study. The exclusion criteria were patients who (1) died during the index hospitalization, (2) discharged themselves against medical advice and refused outpatient follow-up, or (3) were transferred to another acute-care facility (Figure 1).

\section{Data collection and definitions}

Patient electronic medical records were reviewed by two physicians (J.G.J. and J.H.A.). Clinical data included age, gender, comorbidities, vital signs, feeding status, mental status, ambulatory status and laboratory findings. We assessed disease burden using the Charlson comorbidity index (CCI $)^{9}$, which assigns a weighted score to each comorbid condition depending on the risk of 1-year mortality.

Medical Aid beneficiaries were considered to have a lower socioeconomic status than National Health Insurance beneficiaries. The severity of pneumonia was assessed using the pneumonia severity index (PSI) ${ }^{10}$ and CURB-65 score $^{11}$ on day 1 of hospitalization.

Inappropriate initial antibiotic therapy (IIAT) was defined as non-susceptibility to the initially prescribed empirical antibiotic by in vitro antibiotic susceptibility testing.

Treatment failure was defined as clinical deterioration during hospitalization with any of the following: (1) progression of pneumonia on radiographs, (2) respiratory failure, (3) need for mechanical ventilation, (4) hemodynamic instability, or (5) development of a new infection focus ${ }^{12}$. Patients were defined as having decompensation of comorbidity if the medical records suggested exacerbation of one or more comorbidities

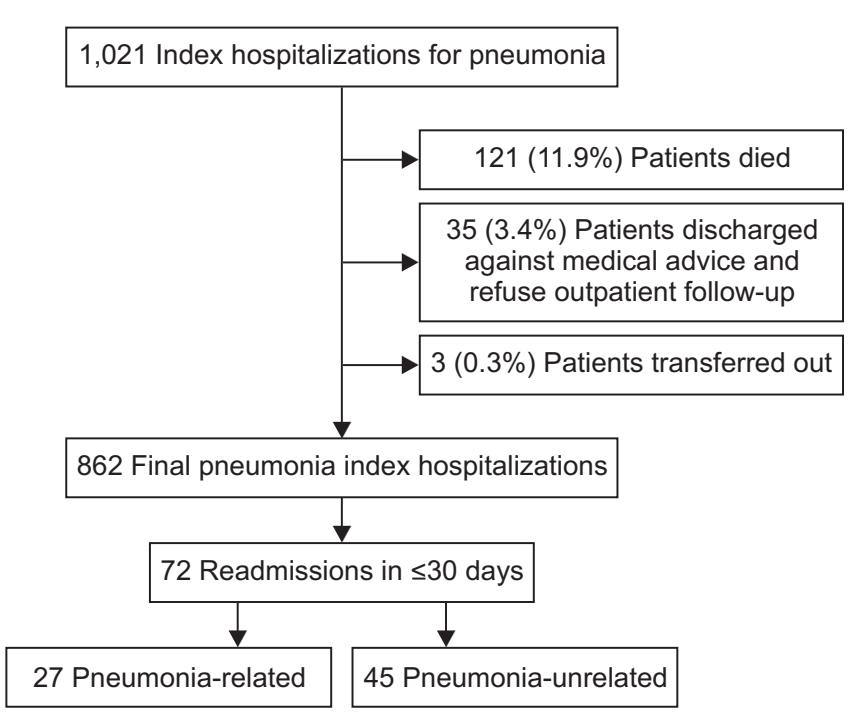

Figure 1. Study design: 1,021 index hospitalizations for communityacquired pneumonia were identified. After excluding patients who died during the index hospitalization, those who discharged themselves against medical advice and refused outpatient follow-up, and those transferred to another acute care facility, the final cohort comprised 862 patients. 
Table 1. Characteristics and outcomes of the patients $(\mathbf{n}=\mathbf{8 6 2})$

\begin{tabular}{|c|c|c|c|c|}
\hline Characteristic & Entire population & Readmission $(n=72)$ & Non-readmission $(n=790)$ & p-value \\
\hline \multicolumn{5}{|l|}{ Host-related } \\
\hline Male sex & $565(65.5)$ & $55(76.4)$ & $510(64.6)$ & 0.043 \\
\hline Age, yr & $68.5 \pm 14.6$ & $72.3 \pm 10.8$ & $68.1 \pm 14.9$ & 0.003 \\
\hline Age $\geq 65 \mathrm{yr}$ & $596(69.1)$ & $60(83.3)$ & $536(67.8)$ & 0.006 \\
\hline \multicolumn{5}{|l|}{ Comorbidities } \\
\hline Congestive heart failure & $64(7.4)$ & $8(11.1)$ & $56(7.1)$ & 0.213 \\
\hline Cerebrovascular disease & $167(19.4)$ & $21(29.2)$ & $146(18.5)$ & 0.028 \\
\hline Dementia & $57(6.6)$ & $6(8.3)$ & $51(6.5)$ & 0.464 \\
\hline Chronic lung disease* & $281(32.6)$ & $38(52.8)$ & $243(30.8)$ & $<0.001$ \\
\hline Connective tissue disease & $16(1.9)$ & $1(1.4)$ & $15(1.9)$ & 1.000 \\
\hline Diabetes mellitus & $182(21.1)$ & $17(23.6)$ & $165(20.9)$ & 0.588 \\
\hline Para/hemiplegia & $64(7.4)$ & $13(18.1)$ & $51(6.5)$ & $<0.001$ \\
\hline Chronic kidney disease & $38(4.4)$ & $7(9.7)$ & $31(3.9)$ & 0.032 \\
\hline Malignancy & $88(10.2)$ & $17(23.6)$ & $71(9.0)$ & $<0.001$ \\
\hline Old myocardial infarction & $30(3.5)$ & $2(2.8)$ & $28(3.5)$ & 1.000 \\
\hline Peripheral vascular disease & $74(8.6)$ & $8(11.1)$ & $66(8.4)$ & 0.424 \\
\hline Liver disease & $25(2.9)$ & $1(1.4)$ & $24(3.0)$ & 0.714 \\
\hline CCI & $1.6 \pm 1.5$ & $2.5 \pm 1.7$ & $1.5 \pm 1.4$ & $<0.001$ \\
\hline $\mathrm{CCI} \geq 1$ & $626(72.6)$ & $69(95.8)$ & $557(70.5)$ & $<0.001$ \\
\hline $\mathrm{CCI} \geq 2$ & $370(42.9)$ & $49(68.1)$ & $321(40.6)$ & $<0.001$ \\
\hline Medical aid & $65(7.5)$ & $6(8.3)$ & $59(7.5)$ & 0.790 \\
\hline Tube feeding $^{\dagger}$ & $55(6.4)$ & $8(11.1)$ & $47(5.9)$ & 0.123 \\
\hline Drowsiness/stupor & $51(5.9)$ & $6(8.3)$ & $45(5.7)$ & 0.428 \\
\hline Nonambulatory status $^{*}$ & $130(15.1)$ & $17(23.6)$ & $113(14.3)$ & 0.035 \\
\hline \multicolumn{5}{|l|}{ Severity } \\
\hline CURB-65 & $1.5 \pm 1.0$ & $1.7 \pm 1.0$ & $1.5 \pm 1.0$ & 0.030 \\
\hline CURB-65 $\geq 3$ & $142(16.5)$ & $17(23.6)$ & $125(15.8)$ & 0.088 \\
\hline PSI & $99.2 \pm 28.4$ & $110.4 \pm 23.6$ & $98.2 \pm 28.5$ & $<0.001$ \\
\hline PSI class $\geq \mathrm{IV}$ & $549(63.7)$ & $58(80.6)$ & $491(62.2)$ & 0.002 \\
\hline ICU admission & $39(4.5)$ & $1(1.4)$ & $38(4.8)$ & 0.244 \\
\hline Mechanical ventilator & $42(4.9)$ & $2(2.8)$ & $40(5.1)$ & 0.569 \\
\hline \multicolumn{5}{|l|}{ Treatment } \\
\hline IIAT & $74(8.6)$ & $7(9.7)$ & $67(8.5)$ & 0.719 \\
\hline \multicolumn{5}{|l|}{ In-hospital evolution } \\
\hline Treatment failure & $72(8.4)$ & $16(22.2)$ & $56(7.1)$ & $<0.001$ \\
\hline Decompensation of comorbidity & $166(19.3)$ & $26(36.1)$ & $140(17.7)$ & $<0.001$ \\
\hline Clinical instability & $0.08 \pm 0.30$ & $0.19 \pm 0.43$ & $0.07 \pm 0.28$ & 0.022 \\
\hline 0 & $796(92.3)$ & $59(81.9)$ & 737 (93.3) & 0.001 \\
\hline$\geq 1$ & $66(7.7)$ & $13(18.1)$ & $53(6.7)$ & \\
\hline Hospital days & $11.6 \pm 12.5$ & $13.8 \pm 9.4$ & $11.4 \pm 12.7$ & 0.867 \\
\hline
\end{tabular}


Table 1. Continued

\begin{tabular}{|c|c|c|c|c|}
\hline Characteristic & Entire population & Readmission $(n=72)$ & Non-readmission $(n=790)$ & p-value \\
\hline \multicolumn{5}{|l|}{ Microorganisms } \\
\hline MDR pathogen & $79(9.2)$ & $7(9.7)$ & $72(9.1)$ & 0.864 \\
\hline MRSA & $21(2.4)$ & $2(2.8)$ & $19(2.4)$ & 0.693 \\
\hline ESBL producing Enterobacteriaceae & $25(2.9)$ & $3(4.2)$ & $22(2.8)$ & 0.457 \\
\hline MDR pseudomonas & $18(2.1)$ & $2(2.8)$ & $16(2.0)$ & 0.657 \\
\hline \multicolumn{5}{|l|}{ Lab } \\
\hline Albumin & $3.3 \pm 0.6$ & $3.1 \pm 0.7$ & $3.3 \pm 0.6$ & 0.008 \\
\hline Lactate & $1.6 \pm 1.1$ & $1.6 \pm 0.8$ & $1.6 \pm 1.1$ & 0.732 \\
\hline NT-ProBNP & $1,020.9 \pm 2,648.9$ & $960.9 \pm 1,446.9$ & $1,026.6 \pm 2,736.1$ & 0.841 \\
\hline CRP & $10.8 \pm 10.1$ & $11.9 \pm 9.1$ & $10.7 \pm 10.2$ & 0.311 \\
\hline Procalcitonin & $3.0 \pm 15.2$ & $1.5 \pm 4.6$ & $3.2 \pm 15.9$ & 0.368 \\
\hline BUN & $17.2 \pm 11.4$ & $18.5 \pm 12.0$ & $17.1 \pm 11.4$ & 0.312 \\
\hline Creatinine & $1.2 \pm 0.8$ & $1.3 \pm 0.8$ & $1.2 \pm 0.8$ & 0.166 \\
\hline Hematocrit & $36.1 \pm 5.8$ & $35.7 \pm 7.6$ & $36.2 \pm 5.6$ & 0.599 \\
\hline Sodium & $136.8 \pm 4.9$ & $135.9 \pm 5.6$ & $136.9 \pm 4.8$ & 0.088 \\
\hline $\mathrm{PaO}_{2} / \mathrm{FiO}_{2}$ ratio & $319.6 \pm 87.7$ & $291.0 \pm 85.8$ & $322.2 \pm 87.5$ & 0.004 \\
\hline $\mathrm{PaO}_{2}$ & $70.5 \pm 20.1$ & $64.6 \pm 16.8$ & $71.0 \pm 20.3$ & 0.010 \\
\hline $\mathrm{PaCO}_{2}$ & $36.8 \pm 21.0$ & $39.5 \pm 30.5$ & $36.6 \pm 19.9$ & 0.257 \\
\hline $\mathrm{pH}$ & $7.4 \pm 0.7$ & $7.4 \pm 0.7$ & $7.4 \pm 0.7$ & 0.615 \\
\hline Platelet & $285.1 \pm 117.7$ & $284.0 \pm 111.1$ & $285.2 \pm 118.4$ & 0.936 \\
\hline Pleural effusion & $140(16.2)$ & $14(19.4)$ & $126(15.9)$ & 0.441 \\
\hline
\end{tabular}

Values are presented as number (\%) or mean \pm SD (range).

*Chronic lung disease includes chronic obstructive pulmonary disease, asthma, bronchiectasis, and interstitial lung disease. ${ }^{\dagger}$ Tube feeding includes nasogastric tube and gastrostomy tube feeding. ${ }^{*}$ Nonambulatory status is defined as using a wheelchair for ambulation or being bedridden.

CCI: Charlson comorbidity index; PSI: pneumonia severity index; ICU: intensive care unit; IIAT: inappropriate initial antibiotic therapy; MDR: multidrug-resistant; MRSA: methicillin-resistant Staphylococcus aureus; ESBL: extended-spectrum beta-lactamase; NT-ProBNP: N-terminal probrain natriuretic peptide; CRP: C-reactive protein; $\mathrm{BUN}$ : blood urea nitrogen; $\mathrm{PaO}_{2}$ : partial pressure of oxygen; $\mathrm{FiO}{ }_{2}$ : fraction of inspired oxygen; $\mathrm{PaCO}_{2}$ : partial pressure of carbon dioxide.

during hospitalization that required intensification of treatment ${ }^{13}$, such as exacerbation of chronic lung disease, acute kidney injury in the presence of chronic kidney disease, or a rapid ventricular response in the form of atrial fibrillation.

We defined clinical instability within 24 hours before hospital discharge using established criteria. A patient with any of the following was considered to be unstable: (1) body temperature $>37.8^{\circ} \mathrm{C}$, (2) respiration rate $>24$ breaths $/ \mathrm{min}$, (3) heart rate $>100$ beats/min, (4) systolic blood pressure $\leq 90$ $\mathrm{mm} \mathrm{Hg}$, or (5) oxyhemoglobin saturation measured by pulse oximetry $<90 \%$ and partial pressure of oxygen in arterial blood $<60 \mathrm{~mm} \mathrm{Hg}^{14}$. Two other criteria used to define clinical instability at hospital discharge (inability to tolerate oral intake and an abnormal mental status) were not available in the medical records.

\section{Outcome variables}

The primary end point was all-cause hospital readmission within 30 days following discharge after the initial hospitalization. Hospital readmission was classified as (1) pneumoniarelated or (2) pneumonia-unrelated readmission.

Pneumonia-related readmission was defined as the presence of (1) radiographic infiltration and (2) acute-onset symptoms suggestive of pneumonia using established criteria ${ }^{13,15}$. Pneumonia-unrelated readmission was defined as the existence of an alternative reason for readmission. Two investigators (J.H.A. and J.G.J.) reviewed the reasons for readmission independently; any discordance was resolved by consensus. 


\section{Statistical analysis}

Continuous variables are expressed as mean \pm standard deviation and were compared by Student's t test or the MannWhitney U test. Categorical variables were compared by chi-squared test or Fisher exact test. Multivariable logistic regression analyses were performed to identify independent risk factors for hospital readmission using variables with a p-value of $<0.1$ in univariable analyses, as measured by the odds ratios (ORs) with 95\% confidence intervals (CIs). A linear-by-linear association test was performed to analyze the 30-day hospital readmission rate according to the number of risk factors. In all analyses, $\mathrm{p}<0.05$ by two-tailed test was considered to indicate statistical significance. All statistical procedures were performed using SPSS software version 21.0 (IBM Corp., Armonk, NY, USA).

\section{Ethical statement}

This study was conducted in accordance with the tenets of the Declaration of Helsinki, and was reviewed and approved by the Institutional Review Board of Yeungnam University Hospital (YUH IRB 2018-06-012). The requirement for informed consent was waived because of the retrospective study design.

\section{Results}

During the study period, 862 patients who survived to hospital discharge were eligible for inclusion and among them 72 (8.4\%) were rehospitalized within 30 days. Pneumonia-related readmission accounted for $37.5 \%$ of the total number of readmissions within 30 days (Figure 1).

\section{Baseline characteristics}

The demographic and baseline characteristics of the patients are presented in Table 1. The mean age of the patients was 68.5 years, and 565 (65.5\%) were males. Patients who were readmitted were older; predominantly male; and more likely to have cerebrovascular disease, chronic lung disease, para/hemiplegia, chronic kidney disease, and malignancy compared with non-readmitted patients. The mean CCI was higher in patients who were readmitted $(2.5 \pm 1.7$ vs. $1.5 \pm 1.4$, $\mathrm{p}<0.001$ ). The frequency of non-ambulatory status (requirement of a wheelchair for ambulation and being bedridden) was higher in readmitted than non-readmitted patients. The CURB-65 ( $1.7 \pm 1.0$ vs. $1.5 \pm 1.0, \mathrm{p}=0.030)$ and PSI ( $110.4 \pm 23.6$ vs. $98.2 \pm 28.5, p<0.001)$ values were significantly higher in patients who were readmitted. With respect to in-hospital evolution, the rates of treatment failure, decompensation of comorbidity, and clinical instability at hospital discharge were significantly higher in readmitted than non-readmitted patients.

\section{Reasons for rehospitalization}

The reasons for rehospitalization were assessed in 72 patients. Pneumonia-related and -unrelated reasons accounted for $37.5 \%$ (3.13\% of the total index hospitalizations) and $62.5 \%$ of the total hospital readmissions, respectively (Table 2).

\section{Factors associated with hospital readmission}

The factors associated with 30-day hospital readmission for pneumonia-related and -unrelated reasons are listed in Tables 3 and 4, respectively. In the multivariable analysis, pneumonia-related readmission was associated with para/ hemiplegia, malignancy, PSI class $\geq 4$ and clinical instability $\geq 1$ at hospital discharge (Table 3). Comorbidities such as chronic

Table 2. Reasons for hospital readmission within 30 days after discharge $(n=72)$

\begin{tabular}{|ll|}
\hline \multicolumn{1}{|c|}{ Reason } & No. (\%) \\
\hline Pneumonia-related $^{*}$ & $27(37.5)$ \\
\hline Pneumonia-unrelated $^{\dagger}$ & $45(62.5)$ \\
\hline Cardiovascular $^{+}$ & $4(5.6)$ \\
\hline Pulmonary & $15(20.8)$ \\
\hline Gastrointestinal & $13(18.1)$ \\
\hline Orthopedic & $1(1.4)$ \\
\hline Neurologic & $1(1.4)$ \\
\hline Renal disorder & $0(0)$ \\
\hline Neoplastic & $2(2.8)$ \\
\hline Endocrine $^{*}$ & $0(0)$ \\
\hline Other $^{*}$ & $9(12.5)$ \\
\hline
\end{tabular}

*Among patients readmitted for pneumonia-related reasons, 12 (44.4\%) were readmitted due to aspiration pneumonia associated with comorbidities. ${ }^{\dagger}$ The pneumonia-unrelated reasons for readmission were as follows: cardiovascular reasons were atrial fibrillation with rapid ventricular response $(n=2)$, acute decompensated heart failure $(n=1)$, and multiple atrial tachycardia due to pulmonary disease $(n=1)$; pulmonary reasons were acute exacerbations of chronic obstructive pulmonary disease $(n=6)$, and idiopathic pulmonary fibrosis $(n=3)$, hemoptysis $(n=2)$, hypoventilation $(n=1)$, pneumothorax $(n=1)$, atelectasis $(n=1)$, and operation for pulmonary causes $(n=1)$; gastrointestinal reasons were gastrointestinal bleeding $(n=5)$, Clostridium difficile-associated diarrhea $(n=3)$, colitis $(n=2)$, peritonitis $(n=1)$, acute appendicitis $(n=1)$, and acute cholangitis $(n=1)$; the orthopedic reason was osteomyelitis of the coccyx $(n=1)$; the neurologic reason was cerebrovascular accident $(n=1)$; the neoplastic reason was lung cancer $(n=2) .{ }^{*}$ Other causes are urinary tract infection $(n=4)$, tracheostomy malfunction $(n=1)$, oral candidiasis $(n=1)$, herpes zoster $(n=1)$, paravertebral infection $(n=1)$, and general weakness with poor oral intake $(n=1)$. 
Table 3. Univariable and multivariable analyses of risk factors for pneumonia-related readmissions among patients with CAP

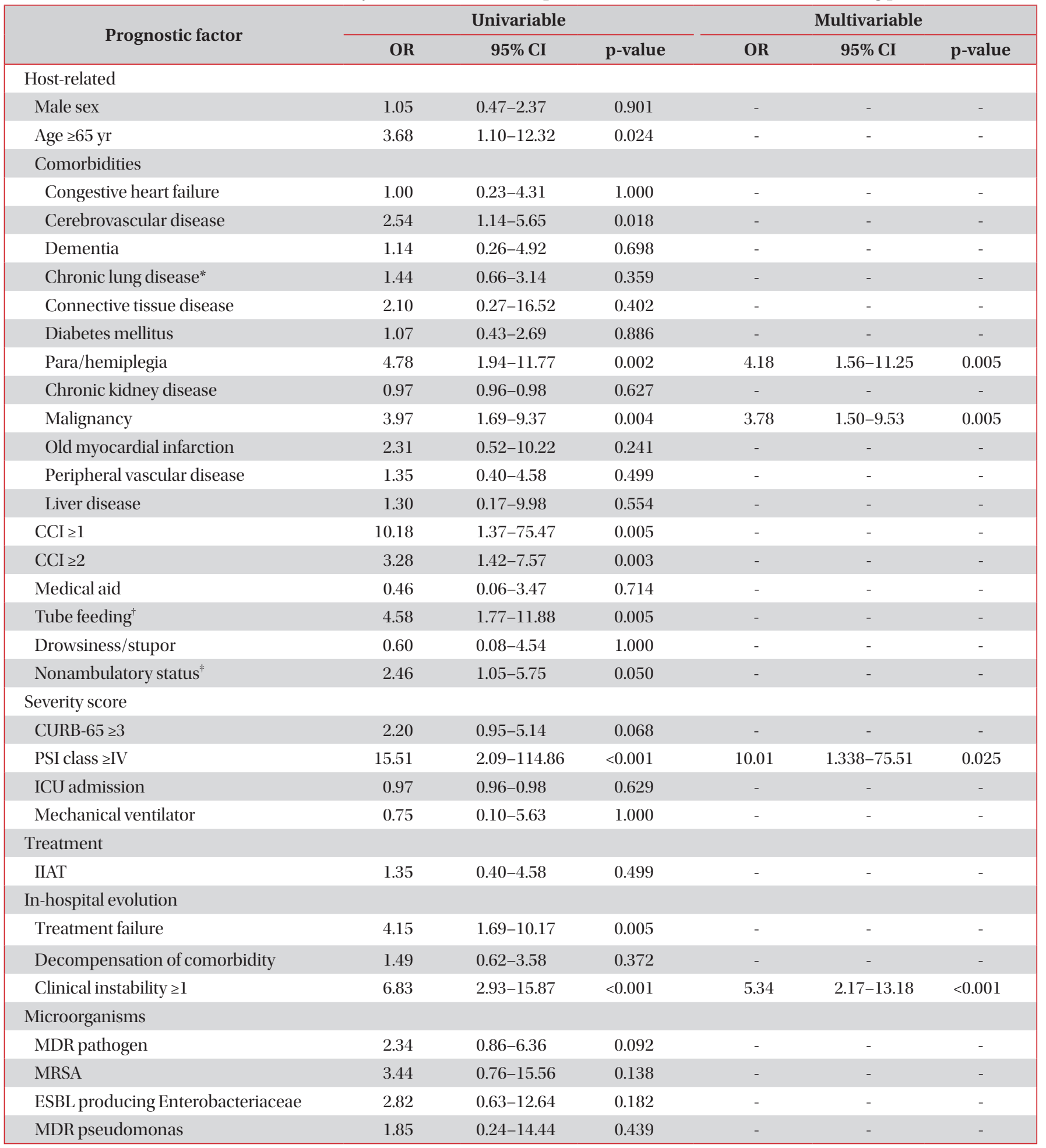

*Chronic lung disease includes chronic obstructive pulmonary disease, asthma, bronchiectasis, and interstitial lung disease. ${ }^{\dagger}$ Tube feeding includes nasogastric tube and gastrostomy tube feeding. ${ }^{*}$ Nonambulatory status is defined as using a wheelchair for ambulation or being bedridden.

CAP: community-acquired pneumonia; OR: odds ratio; CI: confidence interval; CCI: Charlson comorbidity index; PSI: pneumonia severity index; ICU: intensive care unit; IIAT: inappropriate initial antibiotic therapy; MDR: multidrug-resistant; MRSA: methicillin-resistant Staphylococcus aureus; ESBL: extended-spectrum beta-lactamase. 
Table 4. Univariable and multivariable analyses of risk factors for pneumonia-unrelated readmissions among patients with CAP

\begin{tabular}{|c|c|c|c|c|c|c|}
\hline \multirow{2}{*}{ Prognostic factor } & \multicolumn{3}{|c|}{ Univariable } & \multicolumn{3}{|c|}{ Multivariable } \\
\hline & OR & 95\% CI & p-value & OR & 95\% CI & p-value \\
\hline \multicolumn{7}{|l|}{ Host related } \\
\hline Male sex & 2.53 & $1.16-5.51$ & 0.016 & - & - & - \\
\hline Age $\geq 65 \mathrm{yr}$ & 1.84 & $0.87-3.87$ & 0.105 & - & - & - \\
\hline \multicolumn{7}{|l|}{ Comorbidities } \\
\hline Congestive heart failure & 2.01 & $0.82-4.95$ & 0.137 & - & - & - \\
\hline Cerebrovascular disease & 1.37 & $0.68-2.77$ & 0.377 & - & - & - \\
\hline Dementia & 1.41 & $0.49-4.08$ & 0.531 & - & - & - \\
\hline Chronic lung disease* & 3.33 & $1.80-6.15$ & $<0.001$ & 2.81 & $1.33-5.95$ & 0.007 \\
\hline Connective tissue disease & 0.95 & $0.93-0.96$ & 1.000 & - & - & - \\
\hline Diabetes mellitus & 1.22 & $0.61-2.46$ & 0.574 & - & - & - \\
\hline Para/hemiplegia & 2.01 & $0.82-4.95$ & 0.137 & - & - & - \\
\hline Chronic kidney disease & 4.67 & $1.93-11.29$ & 0.002 & 5.70 & $2.21-14.74$ & $<0.001$ \\
\hline Malignancy & 2.34 & $1.09-5.03$ & 0.039 & - & - & - \\
\hline Old myocardial infarction & 0.95 & $0.93-0.96$ & 0.397 & - & - & - \\
\hline Peripheral vascular disease & 1.36 & $0.52-3.55$ & 0.580 & - & - & - \\
\hline Liver disease & 0.95 & $0.93-0.96$ & 0.636 & - & - & - \\
\hline $\mathrm{CCI} \geq 1$ & 8.63 & $2.07-35.91$ & $<0.001$ & - & - & - \\
\hline $\mathrm{CCI} \geq 2$ & 2.81 & $1.49-5.30$ & 0.001 & - & - & - \\
\hline Medical aid & 1.58 & $0.60-4.14$ & 0.377 & - & - & - \\
\hline Tube feeding $^{\dagger}$ & 0.67 & $0.16-2.84$ & 1.000 & - & - & - \\
\hline Drowsiness/stupor & 2.06 & $0.79-5.56$ & 0.180 & - & - & - \\
\hline Nonambulatory status ${ }^{*}$ & 1.44 & $0.68-3.06$ & 0.344 & - & - & - \\
\hline \multicolumn{7}{|l|}{ Severity score } \\
\hline CURB-65 $\geq 3$ & 1.29 & $0.61-2.73$ & 0.512 & - & - & - \\
\hline PSI class $\geq \mathrm{IV}$ & 1.43 & $0.74-2.76$ & 0.288 & - & - & - \\
\hline ICU admission & 0.47 & $0.06-3.47$ & 0.716 & - & - & - \\
\hline Mechanical ventilator & 0.43 & $0.06-3.20$ & 0.719 & - & - & - \\
\hline \multicolumn{7}{|l|}{ Treatment } \\
\hline IIAT & 1.04 & $0.36-3.00$ & 0.790 & - & - & - \\
\hline \multicolumn{7}{|l|}{ In-hospital evolution } \\
\hline Treatment failure & 2.99 & $1.38-6.49$ & 0.009 & 2.78 & $1.22-6.34$ & 0.015 \\
\hline Decompensation of comorbidity & 3.33 & $1.80-6.18$ & $<0.001$ & 2.22 & $1.01-4.67$ & 0.036 \\
\hline Clinical instability $\geq 1$ & 1.19 & $0.41-3.43$ & 0.771 & - & - & - \\
\hline \multicolumn{7}{|l|}{ Microorganisms } \\
\hline MDR pathogen & 0.45 & $0.11-1.88$ & 0.422 & - & - & - \\
\hline MRSA & 0.95 & $0.93-0.96$ & 0.621 & - & - & - \\
\hline ESBL producing Enterobacteriaceae & 0.75 & $0.10-5.68$ & 1.000 & - & - & - \\
\hline MDR pseudomonas & 1.07 & $0.14-8.22$ & 1.000 & - & - & - \\
\hline
\end{tabular}

*Chronic lung disease includes chronic obstructive pulmonary disease, asthma, bronchiectasis, and interstitial lung disease. ${ }^{\dagger}$ Tube feeding includes nasogastric tube and gastrostomy tube feeding. ${ }^{*}$ Nonambulatory status is defined as using a wheelchair for ambulation or being bedridden.

CAP: community-acquired pneumonia; OR: odds ratio; CI: confidence interval; CCI: Charlson comorbidity index; PSI: pneumonia severity index; ICU: intensive care unit; IIAT: inappropriate initial antibiotic therapy; MDR: multidrug-resistant; MRSA: methicillin-resistant Staphylococcus aureus; ESBL: extended-spectrum beta-lactamase. 
lung disease and chronic kidney disease, treatment failure, and decompensation of comorbidities were associated with the pneumonia-unrelated 30-day readmission rate (Table 4).

The likelihood of hospital readmission within 30 days increased with increasing number of risk factors $(\mathrm{p}<0.001$, test for trend) (Figure 2). The 30-day hospital readmission rate after hospital discharge was low $(\leq 3.0 \%)$ in patients with no risk factors. In patients with three risk factors, the 30-day hospital readmission rate was considerable (pneumonia-related OR, 42.86; 95\% CI, 8.53-215.28; pneumonia-unrelated OR, 35.62; 95\% CI, 11.57-109.63).

\section{Discussion}

In this study, of the 862 patients hospitalized for CAP, 72 (8.4\%) were readmitted within 30 days following hospital discharge. Pneumonia-related and -unrelated causes accounted for $37.5 \%$ and $62.5 \%$ of the total readmissions, respectively. The risk factors for pneumonia-related readmission (para/ hemiplegia, malignancy, PSI class $\geq 4$, and clinical instability $\geq 1$ at hospital discharge) were different from those for pneumonia-unrelated readmission (chronic lung disease, chronic kidney disease, treatment failure, and decompensation of comorbidities). Moreover, the likelihood of hospital readmission increased with increasing number of risk factors.

Several studies performed in the United States and Europe have assessed readmission for pneumonia ${ }^{13,15-17}$. In South Korea, the number of physicians per bed is reportedly associated with readmission of elderly CAP patients ${ }^{6}$. However, this study included only patients $>65$ years and did not assess demographic or disease-specific factors. To our knowledge, ours is the first Asian study to evaluate risk factors for hospital readmission following discharge of general adult patients (age $\geq 18$ years) hospitalized for CAP.

The all-cause 30-day readmission rate after hospitalization for pneumonia was $8.4 \%$, compared to $7.3 \%-18.3 \%$ in prior works ${ }^{4,13,18}$. In a systematic chart review, the pooled all-cause

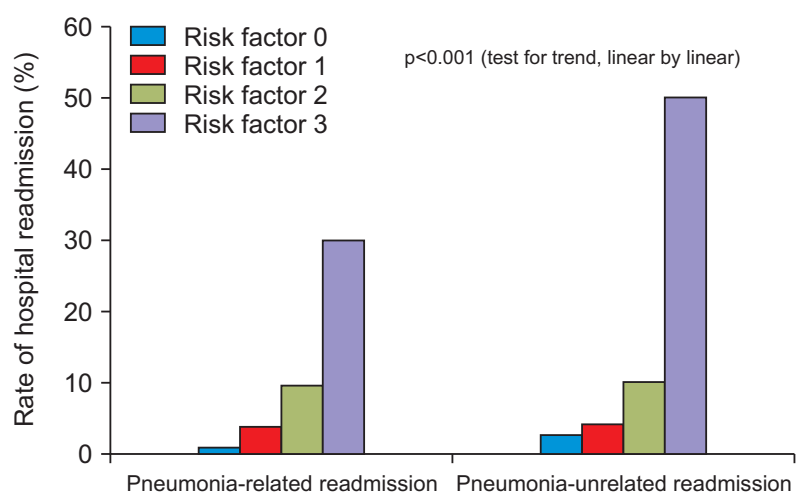

Figure 2. Readmission rate according to number of risk factors. 30-day readmission rate was $11.6 \%$. Generally, US-based studies and those involving elderly patients (age $\geq 65$ years) show higher rates of all-cause 30-day readmission than non-USbased studies and those involving adults (age $\geq 18$ years) ${ }^{19}$. The all-cause 30-day readmission rate in our study was similar to the values reported by non-US-based studies and those not restricted to elderly patients $\mathrm{s}^{13,18}$. A prior work involving elderly patients (age $>65$ years) in South Korea reported a higher (19.1\%) all-cause 30-day readmission rate than our finding ${ }^{6}$. Thus, the healthcare environment (including hospital accessibility, financial issues, and medical practices) and age are important determinants of readmission rates.

The leading causes of 30-day readmission in our study were pneumonia $(37.5 \%)$, pulmonary disease $(20.8 \%)$, gastrointestinal disease (18.1\%), and cardiovascular disease (5.6\%). Pneumonia, heart failure/cardiovascular disease, and chronic obstructive pulmonary disease/pulmonary disease are the leading reasons for hospital readmission within 30 days ${ }^{19}$. Interestingly, gastrointestinal causes were the third-mostfrequent reasons for hospital readmission. This is higher than in previous reports, possibly because of the effect of the large proportion $(63.7 \%)$ of high-risk (PSI class $\geq 4$ ) patients on the incidence of gastrointestinal bleeding and Clostridium difficile-associated diarrhea (CDAD) (8 cases/all 13 gastrointestinal causes) ${ }^{13,15,18}$. Indeed, seven of the eight patients (87.5\%) who developed gastrointestinal bleeding and CDAD were in the high-risk group (PSI class $\geq 4$ ).

Para/hemiplegia and malignancy were associated with pneumonia-related readmission. This may be because patients with these comorbidities have decreased performance status and/or immunity. Among patients readmitted for pneumonia-related reasons, $44.4 \%(12 / 27)$ had aspiration pneumonia associated with comorbidities. Indeed, the aspiration risk is reportedly associated with higher rates of readmission and recurrent pneumonia in patients with $\mathrm{CAP}^{20}$. Thus, evidencebased interventions, such as angiotensin-converting enzyme inhibitors ${ }^{21,22}$, could prevent readmission for recurrent aspiration pneumonia in high-risk patients. Moreover, maintaining good oral hygiene, withholding unnecessary medications, food thickening, sitting upright while eating, head elevation of at least $30^{\circ}$, and swallowing rehabilitation might decrease the risk of aspiration in high-risk patients ${ }^{23}$. Education of patients regarding these interventions before hospital discharge would decrease the pneumonia-related readmission rate.

PSI class $\geq I V$ and clinical instability at hospital discharge were risk factors for pneumonia-related readmission. Unlike previous studies ${ }^{15,16,18}$, our findings revealed that PSI class $\geq I V$ is an independent risk factor for pneumonia-related readmission. However, those prior works did not separate pneumoniarelated and -unrelated readmissions. We found that a clinical instability score of $\geq 1$ was an independent risk factor for readmission, in accordance with previous reports ${ }^{13,17}$. Discharge at the optimal time (taking into consideration clinical stability) 
could prevent pneumonia-related readmission, especially in high-risk patients (PSI class $\geq \mathrm{IV}$ ). Despite clinical instability, there may be concerns about being discharged from the hospital in this study. However, it is thought that there might have been cases of stable conditions that could be discharged clinically but belonging to clinical instabilities defined in our study. In addition, many patients transferred to secondary hospitals due to comorbidities, so it is thought that there have been cases of step down referral with some clinical instability. Neither the presence of a multidrug-resistant (MDR) pathogen nor IIAT was associated with pneumonia-related readmission.

We found that comorbidities such as chronic lung disease and chronic kidney disease, treatment failure, and decompensation of comorbidities (as in a prior report ${ }^{13}$ ) were associated with pneumonia-unrelated readmission. This is because pneumonia promotes inflammation and dysregulates the immune response, leading to progression of comorbidities. Also, decompensation of comorbidities during hospitalization doesn't recover well even after being discharged from the hospital, leading to the possibility of rehospitalization. In particular, the proportion of patient with chronic lung disease in our study is high, the acute exacerbation of chronic lung disease caused by pneumonia is thought to have influenced on rehospitalization. Disease severity, presence of an MDR pathogen, and IIAT were not associated with pneumonia-unrelated readmission.

This study had several limitations. First, because it was a retrospective study conducted at a single center, the results cannot be generalized. Second, we did not assess readmissions to other hospitals; we attempted to overcome this limitation by excluding patients who refused outpatient follow-up. However, the number of patients who required readmission could have been underestimated. We believe that a multicenter, prospective study is required in order to assess the causes of readmission in patients with pneumonia. Third, we focused on patient-related factors as determinants of readmission. Physicians' admission decisions and healthcare-system factors are associated with hospital readmissions but were not investigated because of a lack of information. Fourth, the investigation of patients who died within 30 days after discharge was difficult, and there was lack of analysis.

In conclusion, we found that $8.4 \%$ of the patients hospitalized for CAP were rehospitalized within 30 days of discharge. This is generally consistent with previous studies involving different populations, regions, and methodologies. Pneumoniarelated and -unrelated causes accounted for $37.5 \%$ and $62.5 \%$, respectively, of the total readmissions. The risk factors for pneumonia-related and -unrelated readmission were different. Strategies to prevent aspiration and to achieve clinical stability at hospital discharge, especially in patients with PSI class $\geq \mathrm{IV}$, para/hemiplegia, or malignancy could prevent pneumonia-related readmission to hospital. Appropriate management of comorbidities in high-risk patients (i.e., those with chronic lung disease and chronic kidney disease) would reduce the pneumonia-unrelated readmission rate. Therefore, aspiration prevention, discharge at the optimal time, and close monitoring of comorbidities may reduce the frequency of readmission among patients with CAP.

\section{Authors' Contributions}

Conceptualization: Ahn JH. Methodology: Jang JG, Ahn JH. Formal analysis: Jang JG, Ahn JH. Writing - original draft preparation: Jang JG. Writing - review and editing: Ahn JH. Approval of final manuscript: all authors.

\section{Conflicts of Interest}

No potential conflict of interest relevant to this article was reported.

\section{Acknowledgments}

We would like to express our appreciation to Prof. Eun Young Choi for proving the data. Her guidance and data was very helpful bringing this work.

\section{Funding}

This work was supported by the Yeungnam University Research Fund in 2019.

\section{References}

1. Mandell LA, Wunderink RG, Anzueto A, Bartlett JG, Campbell GD, Dean NC, et al. Infectious Diseases Society of America/ American Thoracic Society consensus guidelines on the management of community-acquired pneumonia in adults. Clin Infect Dis 2007;44 Suppl 2:S27-72.

2. Shin HY, Lee JY, Song J, Lee S, Lee J, Lim B, et al. Cause-ofdeath statistics in the Republic of Korea, 2014. J Korean Med Assoc 2016;59:221-32.

3. Choi MJ, Song JY, Noh JY, Yoon JG, Lee SN, Heo JY, et al. Disease burden of hospitalized community-acquired pneumonia in South Korea: analysis based on age and underlying medical conditions. Medicine (Baltimore) 2017;96:e8429.

4. Dharmarajan K, Hsieh AF, Lin Z, Bueno H, Ross JS, Horwitz LI, et al. Diagnoses and timing of 30-day readmissions after hospitalization for heart failure, acute myocardial infarction, or pneumonia. JAMA 2013;309:355-63.

5. Dharmarajan K, Wang Y, Lin Z, Normand ST, Ross JS, Horwitz 
LI, et al. Association of changing hospital readmission rates with mortality rates after hospital discharge. JAMA 2017;318: 270-8.

6. Lee JE, Kim TH, Cho KH, Han KT, Park EC. The association between number of doctors per bed and readmission of elderly patients with pneumonia in South Korea. BMC Health Serv Res 2017;17:393.

7. De Alba I, Amin A. Pneumonia readmissions: risk factors and implications. Ochsner J 2014;14:649-54.

8. Ahn JH, Choi EY. Expanded A-DROP score: a new scoring system for the prediction of mortality in hospitalized patients with community-acquired pneumonia. Sci Rep 2018;8:14588.

9. Charlson ME, Sax FL, MacKenzie CR, Fields SD, Braham RL, Douglas RG Jr. Assessing illness severity: does clinical judgment work? J Chronic Dis 1986;39:439-52.

10. Fine MJ, Auble TE, Yealy DM, Hanusa BH, Weissfeld LA, Singer DE, et al. A prediction rule to identify low-risk patients with community-acquired pneumonia. N Engl J Med 1997;336: 243-50.

11. Lim WS, van der Eerden MM, Laing R, Boersma WG, Karalus $\mathrm{N}$, Town GI, et al. Defining community acquired pneumonia severity on presentation to hospital: an international derivation and validation study. Thorax 2003;58:377-82.

12. Yandiola PPE, Capelastegui A, Quintana J, Diez R, Gorordo I, Bilbao A, et al. Prospective comparison of severity scores for predicting clinically relevant outcomes for patients hospitalized with community-acquired pneumonia. Chest 2009;135: 1572-9.

13. Capelastegui A, Espana Yandiola PP, Quintana JM, Bilbao A, Diez R, Pascual S, et al. Predictors of short-term rehospitalization following discharge of patients hospitalized with community-acquired pneumonia. Chest 2009;136:1079-85.

14. Halm EA, Fine MJ, Marrie TJ, Coley CM, Kapoor WN, Obrosky DS, et al. Time to clinical stability in patients hospitalized with community-acquired pneumonia: implications for practice guidelines. JAMA 1998;279:1452-7.

15. Jasti H, Mortensen EM, Obrosky DS, Kapoor WN, Fine MJ. Causes and risk factors for rehospitalization of patients hospitalized with community-acquired pneumonia. Clin Infect Dis 2008;46:550-6.

16. Shorr AF, Zilberberg MD, Reichley R, Kan J, Hoban A, Hoffman J, et al. Readmission following hospitalization for pneumonia: the impact of pneumonia type and its implication for hospitals. Clin Infect Dis 2013;57:362-7.

17. Halm EA, Fine MJ, Kapoor WN, Singer DE, Marrie TJ, Siu AL. Instability on hospital discharge and the risk of adverse outcomes in patients with pneumonia. Arch Intern Med 2002; 162:1278-84.

18. Adamuz J, Viasus D, Camprecios-Rodriguez P, CanavateJurado O, Jimenez-Martinez E, Isla P, et al. A prospective cohort study of healthcare visits and rehospitalizations after discharge of patients with community-acquired pneumonia. Respirology 2011;16:1119-26.

19. Prescott HC, Sjoding MW, Iwashyna TJ. Diagnoses of early and late readmissions after hospitalization for pneumonia: a systematic review. Ann Am Thorac Soc 2014;11:1091-100.

20. Komiya K, Rubin BK, Kadota JI, Mukae H, Akaba T, Moro H, et al. Prognostic implications of aspiration pneumonia in patients with community acquired pneumonia: a systematic review with meta-analysis. Sci Rep 2016;6:38097.

21. Harada J, Sekizawa K. Angiotensin-converting enzyme inhibitors and pneumonia in elderly patients with intracerebral hemorrhage. J Am Geriatr Soc 2006;54:175-6.

22. Caldeira D, Alarcao J, Vaz-Carneiro A, Costa J. Risk of pneumonia associated with use of angiotensin converting enzyme inhibitors and angiotensin receptor blockers: systematic review and meta-analysis. BMJ 2012;345:e4260.

23. Luk JK, Chan DK. Preventing aspiration pneumonia in older people: do we have the 'know-how'? Hong Kong Med J 2014; 20:421-7. 